\title{
Le monde arabe en filigrane dans l'œuvre de Tahar Ben Jelloun
}

\author{
Virginia Boza Araya'
}

Universidad Nacional de Costa Rica

\section{RESUMEN}

Tahar Ben Jelloun es un escritor marroquí de expresión francesa; sin embargo, su obra está marcada tanto en la forma como en el contenido por la cultura árabe. Desde el nombre de sus novelas el autor clama su origen musulmán. En sus obras el novelista reconstituye las callejuelas de sus medinas por medio de una estructura laberíntica en la que la cultura arabo-musulmana cobra vida gracias a un vocabulario denotativo y connotativo. El autor describe la sociedad tradicional y moderna con lujo de detalles denotándolas y connotándolas por medio de las acciones, los gestos y los intercambios discursivos de sus personajes. Las referencias al Islam como parte integrante de su identidad, a la literatura, a la caligrafía, a los grandes filósofos permiten al lector comprender me jor la religión y las creencias de la sociedad arabomusulmana.

\section{RÉSUMÉ}

Tahar Ben Jelloun est un écrivain marocain d'expression française cependant son œuvre reste foncièrement arabe autant dans la forme que dans le fond. En effet la structure de ses romans emprunte les dédales de ses médinas et la présence de la culture arabo-musulmane y est indiscutable. Dès les titres de ses romans l' auteur confirme son appartenance au monde musulman soit par les vocables choisis soit par leur sonorité. L'auteur dépeint la société traditionnelle et la société moderne grâce à un réseau de références tant dénotatives que connotatives grâce aux actions, aux gestes, aux échanges

$\overline{1}$ Correo electrónico: dantrou@hotmail.com 
discursif de ses personnages, aux situations décrites. Les allusions à l'Islam comme une partie indissociable de l'identité, à la littérature, à la calligraphie, aux grands philosophes permettent au lecteur de mieux comprendre la religion et les croyances de la société arabo-musulmane.

Palabras clave: literatura de expresión francesa, cultura magrebina, cultura arabo-musulmana, mundo árabe, lengua je connotativo, lengua je denotativo. Mots-clés : littérature francophone, culture maghrébine, culture arabomusulmane, monde arabe, langage connotatif, langage dénotatif.

Doit-on étiqueter de littérature française toute production littéraire en français quel que soit son origine ? Cette question a pendant longtemps déclenché une polémique dans le monde francophone. Il est évident que l'origine des écrivains, leur vécu, leur histoire impose une spécificité. Les littératures d'expression française sont toutes marquées par l'altérité que leur histoire coloniale leur a imposée. En parlant du roman maghrébin Charles Bonn affirme que : «Le roman maghrébin, et même la littérature arabe, $s$ 'inscrivent sous le signe de l'altérité, de la double culture $»^{2}$.

Le roman maghrébin est « l'œuvre d' un Maghrébin qui écrit tout simplement comme romancier algérien, marocain, tunisien $»^{3}$. Il est jalonné par un réseau de références, d'allusions, d'images propres au monde arabe doublé d'un implicite culturel ancré dans sa mémoire. Ce dédale référentiel est tout d'abord dénotatif. Il fait référence à la matérialité du monde arabo-musulman (noms propres, objets de la vie quotidienne, vêtements, noms définissant l'espace géographique entre autres). Il est doublé par un message connotatif ${ }^{4}$ révélateur des conceptions et des desseins de l'auteur. On peut ajouter à ce réseau ce

$\overline{2}$ Charles Bonn, Au miroir de l'autre (Paris: L'Harmattan, 1991) 16.

${ }^{3}$ Jean Dejeux. Lalittérature maghrébine d'expression française (Paris : Presses Universitaires Françaises, 1992).

${ }^{4}$ Un logiciel a été spécialement conçu afin de déterminer le nombre d'occurrences de ce langage dénotatif et connotatif ce qui permettra de déduire l'univers mental de l'auteur et les principaux traits de la société arabo-musulmane. 
que Robert Galisson ${ }^{5}$ définit comme vocabulaire « à charge culturel partagée ». L'étude du langage à charge culturelle arabe facilite la compréhension de l'univers mental de cette culture qui est empreint de mystère.

Dès les titres de ses romans, l'auteur confirme son appartenance au monde musulman soit par les vocables choisis soit par leur sonorité. La Prière de l'absent et La nuit sacrée évoquent, tous les deux, la religion islamique, composante indissociable de la culture maghrébine. La Prière de l'absent est la traduction de l'une des prières réservées aux morts dans le monde musulman ${ }^{6}$. Dans son œuvre, l'absent est celui qui a perdu son identité à cause de la colonisation, celui qui a oublié ses origines et en est mort. Une mort évidemment symbolique. La nuit sacrée correspond à « la vingt-septième du mois de ramadan, nuit de la « descente » du Livre de la communauté musulmane »". Le vocable "sable» du titre L'Enfant de sable fait référence par métonymie au désert et donc connote également l'espace physique arabe. Par contre, dans Moha le fou, Moha le sage c'est la sonorité du prénom « Moha » qui impose cet univers à l'imagerie du lecteur.

Ce système langagier à charge culturelle arabe peut être classé en différentes catégories.

\section{Lexique}

Tout au long des romans l' auteur bâtit le cadre spatial grâce à un foisonnement de noms propres de villes, de villages, de régions, de sites historiques ou revêtant une connotation religieuse ${ }^{8}$. En général, ses personnages parcourent le pays à la recherche d'une identité

\footnotetext{
5 Robert Gallison, « Culture et lexiculture partagées : les mots comme lieux d'observation des faits culturels » in Etudes de linguistique appliquée : Observer et décrire les faits culturels (1988) 69.

${ }^{6}$ Boucheikri, Commentaire (<http://www.lisons.info/Ben-Jelloun-Tahar-auteur-22.php>, 2005).

7 Tahar Ben Jelloun, La nuit sacrée (Paris : Editions Club France Loisirs, 1987) 23.

${ }^{8}$ Fès Jedid-3, Fès-17, Marrakech-30, Meknès-10, Casablanca-11, Sef rou-3.
} 
perdue. Ahmed, le personnage du diptyque Ahmed/Zahra, réalise une sorte de pèlerinage qui le conduit à Marrakech. Il souhaite eff acer son passé qu'il a vécu sous l' apparence d'un homme par la volonté du père. Celui-ci n'avait pas d'héritier mâle ce qui pose problème dans les sociétés arabo-musulmanes. A la naissance du héros, le père décide de l'élever comme un homme afin de récupérer son honneur perdu. A la mort du père, Ahmed part à la recherche de sa vraie identité, celle d'une femme. Il déambule dans le pays jusqu' à Marrakech :

... qu'il fallait continuer le voyage jusqu'à Tétouan, jusqu'à Fès et Marrakech. Cette visite a quelque chose du pèlerinage. Je dois accomplir cela sans m'arrêter jusqu'à redonner à cette âme la paix, la sérénité et le silence dont elle a besoin?

Le voyage initiatique accompli par les protagonistes de $L a$ Prière de l'absent permet au lecteur de découvrir les villes avec leurs spécificités, leurs couleurs, leur atmosphère. Ces personnages traversent les villes impériales de Fès, Meknès, Marrakech mais aussi Casablanca, Rabat Hodh, Tanger, Khémisset qui sont plus modernes :

Fès Jedid, la médina est à droite ...les maisons petites et imbriquées les une... On voit la Qaraouiyine, éternelle dans le silence de ce matin sublime, un peu plus à droite le mausolée de Moulay Idriss, tache verte des tuiles alignées. Des terrasses vides. Des petits minarets de quartier. Au fond, une colline rouge et un ciel d'un bleu insupportable....

Fès, creuset d'une civilisation et d'une culture $!^{10}$

Ils transitent par des villages tels que Zarhoun, Saquiat el Hamra, Asilah, Sefrou, Smara, Tiznit, Tétouan, Salé, Khémisset, Azrou, Koutoubia, Sidi Harazem, Taza, Ain Leuh, Mellah, Dakhla, Adrar,

\footnotetext{
$\overline{9}$ Tahar Ben Jelloun, L'Enfant de sable (Editions : France Loisir, 1985) 182.

10 Tahar Ben Jelloun, La Prière de l'absent (Paris : Editions du Seuil, 1981) 56, 84.
} 
Haouz Tarfaya, Sahara ; des oueds : l'oued Noun, Tindouf, l'oued Noun à Adrar et Tiris, Douar Doum, Bou Khrarab (une rivière étroite) des quartiers : Médina, Beni Makada, la Qissaria, Fès Jedid. Dans les villes et les villages ils parcourent des rues: Borj Dhab, Bab el Khokha, traversent des ponts : Rsif, visitent des monastères : Tioumililine, des derbs, des mosquées d'El Habous, Ahl Barakat et des gares routières : Jamaa el Fna entre autres, passent la nuit dans les mosquées, visitent des mausolées.

L'auteur insiste sur le côté traditionnel : les médinas, les souks, les places publiques qui revêtent une importance capitale dans la culture arabe. En effet, la place publique constitue un carrefour qui favorise la rencontre des cultures, les échanges interculturels entre les différentes ethnies qui composent la population marocaine. Une mosaïque de peuples s'y côtoient : des Berbères (17) (premiers habitants de l'Afrique du Nord), des Kabyles (2) (Berbères d'Algérie), des hommes bleus (1) (nomades du Sahara), des Arabes (5) ${ }^{11}$, des Gourna, des Aroubia, des Jbala, des Reguibets, des Marrakchi, des Chorfas du Sud, et des Filaliens. Ces places sont également les gardiennes de la tradition car c'est le lieu de rencontre des conteurs avec leurs auditeurs. Ils perpétuent ainsi les traditions orales et le folklore arabo-musulman.

D'autres noms propres introduisent, dans les récits, les héros de l'histoire du Maroc, ceux qui ont fait le prestige de leur culture : hommes de courage et possédant les vertus de l'Islam. C'est le cas du cheïkh Muhammad Mustafa Ma-al-Aynayn (28 occurrences) du Sud qui a organisé la résistance contre les français suivi plus tard par l'émir Abdelkrim al Khattabi (2) du nord. Ils évoquent également ceux qui ont trahi le pays au moment de la colonisation et pendant la période du protectorat, le Pacha El Glaoui (1) entre autres. «Le pays était ainsi tendu dans, le temps, dans l'histoire, entre deux mémoires illustres ${ }^{12}$.

\footnotetext{
"Trois occurrences pour langue arabe et trois en tant qu'adjectif pour définir l'art et l'architecture.

12 La Prière ..., 138.
} 
D' autres grands hommes cités dans les romans sont Mohammed Fadil Wult Mamin, chef de la Qadiriya, la confrérie du Destin, père du héros sudiste de la résistance, Moulay Idriss (7 occurrences) : fondateur de Fès qui est devenu saint par la suite, Sidi Mohammed (3) Allal Ben Abdallah, résistant, des sultans célèbres : Arafa (1), Abdel Aziz (3), Moulay Hassan (1), Moulay Hafid (2), des émirs Ould Aida (2). Tous ces noms propres ont des connotations politiques et historiques. D'autres illustrent l'art, la littérature (Abû Nawâss - poète, Abû Hayyân Tawhîdî -, Al Hallaj, Hasan Basri, Ghazâli, Ibn 'Arabi mystiques, Na't al-bidayat - oeuvre mystique), la musique (Oum Khalthoum grande chanteuse) et des personnages de légende ${ }^{13}$ qui hantent la mémoire et confortent les superstitions du peuple. Le peuple n'y est pas absent, des prénoms ${ }^{14}$ comme Favila, Malika, Lalla, Rhadia, Ahmed, Zahra, Assise, Argane, Riha, Nedjma, Aïcha, Abbas, Abdallah, Driss, Salah, Suleiman, Hammou, Jamal, Qamar parsèment les pages les colorant de leurs sonorités arabes et, certains d'entre eux, comportent une connotation religieuse. En effet, bon nombre de garçons comme Mohammed Mokhtar, héros de La Prière de l'absent, porte le prénom du Prophète Mohammed.

Par petites touches, l'auteur dépeint la société et la culture arabe en utilisant des arabismes définissant la vie quotidienne, les vêtements, l'alimentation, la religion. Etant donné l'immigration arabe, assez importante en France, bon nombre de substantifs a été assimilé et même inclus dans les dictionnaires ${ }^{15}$. Parmi les arabismes insérés dans les dictionnaires français ont compte : dirham (monnaie), hammam (bain public), kohol (fard à paupières), kif (mélange de chanvre indien et de tabac), haschisch (drogue). En cuisine, l'un des plus connus est le couscous mais aussi le tajine et des épices comme la harissa. D'autres substantifs concernant des vêtements ont également été adoptés : le tarbouche et le fez (coiffures), le haïk (pièce de tissus), la

\footnotetext{
13 Aïcha Kandisha. C'était l'ogresse traditionnelle, Ghoula, Mina-la-Chauve, l'oeil de l'Éclair, BentMqabar, Fiancée-de-la-Mer. Hamqat-Njum. Gazelle-des-Ténèbres...Antar, Mahdi, Aicha-la-Comtesse.
} 
djellaba (robe longue), le burnous (manteau), séroual (pantalon) et dans le domaine du pouvoir: des titres tels qu'émir, sultan, cheikh, pacha comportant une connotation politique.

La plupart des vocables du champ sémantique de l'Islam est connu ${ }^{16}$ en français et répertorié dans les dictionnaires. Ces vocables foisonnent dans l'œuvre de Ben Jelloun. Les mots les plus usités par ordre d'occurrences sont : Prophète (54), Coran (40), Islam (29), Marabout (29), La Mecque (23), Allah (20), verset (19), musulman (19), sourate (9), muezzin (7), ramadan (7), minarets (6), imam (5). Il est intéressant de remarquer que les mots français prière (99), linceul (25) et voile (15), bien qu'ils ne soient pas d'origine arabe, sont déterminants dans ce domaine car ils dénotent les pratiques religieuses et connotent l'idée d'une foi profonde. Les vocables les plus utilisés réfèrent aux symboles de cette religion : Le prophète, le livre sacré et ses sourates, les lieux sacrés et les lieux de pratique religieuse. N'oublions pas Ulama (interprète du Coran), minbar (chaire à prêcher d'une mosquée), mihrab (niche à l'intérieur d'une mosquée), zaouïa (établissement religieux).

D'autres substantifs n'ont pas encore étè lexicalisés. Ils sont moins usités dans un milieu francophone. De ce fait, le lecteur qui n' est pas familiarisé avec la langue arabe, ne peut pas saisir leurs sens dénotatif. Pour palier ce manque, l'écrivain énonce le sens par une phrase explicative qu'il met entre parenthèse : « des lassourtis (on désignait ainsi les policiers en civil, des Arabes qui collaboraient avec les services de renseignements généraux français) ${ }^{17}$.

Parfois l'auteur insère le mot dans un contexte qui permet au lecteur de déduire le sens. Lorsqu'il évoque, par exemple, les vocables

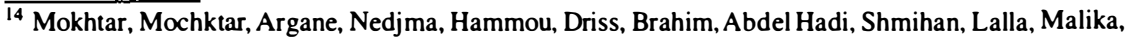
Aïcha, Moha, Amar, Fatouma, Salem, Om Abbas, Bouchaib, Favila, Zineb, Rhadia, Hadj, Ahmed, Zahra, El Hadj, Si Suleiman.

${ }^{15}$ Bourkis Ridha, Tahar Ben Jelloun. La poussièred'or et la face masquée. Approche linguistique (Paris : L'Harmattan, 1995) 133.

${ }^{16}$ Cependant il en reste qui ne sont pas lexicalisés : Fatiha (la sourate de l'ouverture), Latif : « Ya Latif/ Ya Latif! „C'était l'appel des musulmans à la bonté d'Allah », fqilı : jeune religieux entre autres.

${ }^{17}$ Ridha, 29.'
} 
djnouns $^{18}$ et djinns il les associe à l'adjectif "possédés », à des substantifs tels que " magie ${ }^{19}$, ou à des expressions métaphoriques " lointains abîmes » qui connotent mystère ; enfer ce qui emmène le lecteur à inférer qu'il s' agit d'esprits malfaisants. L'ensemble de mots, ainsi formés, comporte une connotation de « fantastique » arabe.

L'auteur peut tout simplement donner leur signification : « Ce sont les mala' ika, les anges de l' au-delà » ou « mahia, une eau de vie à base de figue ${ }^{20}$ ou khammas, les ouvriers. Il donne également des explications en note en bas de page ${ }^{21}$. Ces informations concernent, entre autres, des mots relatifs au sexe émis par les femmes dans le hammam : mani « sperme », glaoui " couilles", taboun « vagin ». Dans cette société phallocrate, les femmes ne peuvent pas parler de sexe librement. Donc lorsqu'elles se retrouvent dans l'intimité du hammam elles en parlent, se libèrent des contraintes que le mariage impose. Outre ces termes à connotation sexuelle, il en existe d'autres qui dénotent des métiers: goumiers et lassourtis (connotation militaire, pouvoir), khammas (ouvriers); des termes culinaires « mahia », « harira » et des plantes entre autres : «fenjel », « ma'joun ».

Dans le lexique à charge culturelle partagée par les marocains en particulier, se trouve les vocables Nord (12)/Sud (12). Ils revêtent une importance capitale car ils évoquent des moments importants de leur histoire. En effet, l'un des événements marquants est l'invasion occidentale et celle-ci est arrivée par le Nord. La résistance, elle s'est organisée dans le Sud. Le Nord ne s'est joint à celle-ci que bien plus tard. Les grands héros de l'histoire sont originaires du Sud. D'autres mots, en relation avec l'univers minéral du désert, évoquent irrémédiablement pour eux les origines : sable (16), désert (10), avec lumière (10), source (10), vertu (7), origine (6), ciel (6) : «C'est un privilège que je suis chargée de confirmer en t'emmenant vers

\footnotetext{
18 Ridha, 203.

19 Tahar Ben Jelloun, Moha le fou, Moha le sage (Paris : Editions du Seuil, 1978) 32.

${ }^{20}$ La Prière ..., 64.

${ }^{21}$ La Prière ..., 31.
} 
l'origine, vers les dunes du Sud, là où tu trouveras des racines et une histoire ${ }^{22}$.

\section{La calligraphie arabe}

Les romans de Tahar Ben Jelloun sont certes écrits en français mais ils n'en restent pas moins l'expression d'une culture arabe riche en traditions, en art, en littérature. Pour lui donner vie à travers ses romans, il utilise une série de techniques qui lui permettent d'imposer l'image d'une culture arabo-musulmane. Outre l'insertion d'un langage dénotatif et connotatif propre à cette culture, il parsème son récit d'invocations, de versets, de sourates, de mots écrits en calligraphie arabe. Ceux-ci sont, en général, liés à la religion islamique, à la philosophie, au mysticisme, rappellent des moments importants de l'histoire du pays ou dénoncent les travers de la société. Ces inscriptions en calligraphie arabe sont transcrites en français pour qu'elles puissent être comprises par tout lecteur non averti. Elles accompagnent les moments importants de la vie des musulmans : la naissance, la mort, l'indépendance entre autres.

Une première catégorie concerne des prières berçant les accouchements et les enterrements. Dans La Prière de l'absent, une série d'invocations sont répétées comme une sorte de litanie afin de soulager les douleurs de la femme qui est en train d'enfanter ${ }^{23}$. Ces litanies invoquent Dieu et les anges pour qu'ils aident la femme à accoucher.

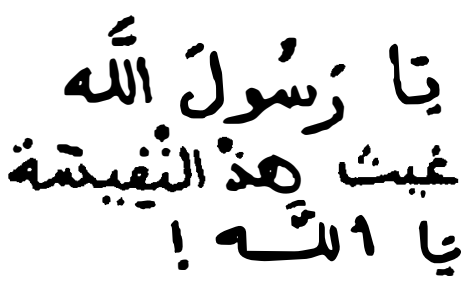

Ô Envoyé de Dieu

Viens au secours de cette femme enceinte

Ô mon Dieu !

22 Ben Jelloun, La Prière ...

${ }^{23}$ La Prière de l'absent, 25-26. 
D'autres prières accompagnent le défunt dans sa dernière demeure lors de ses funérailles.

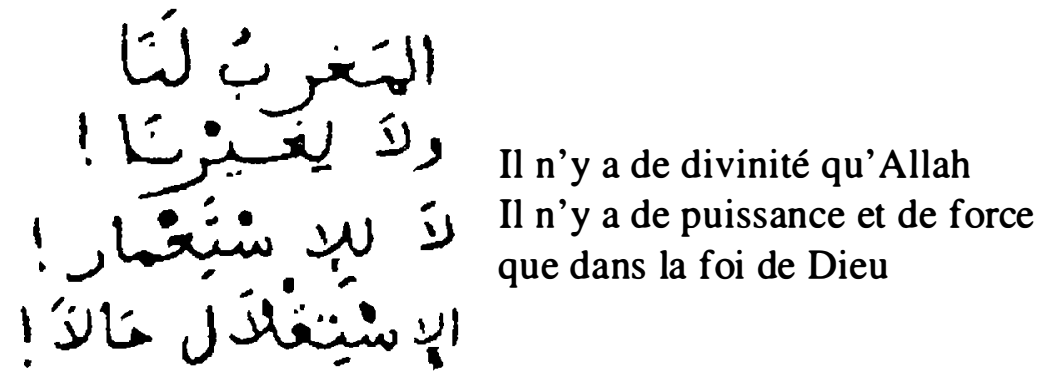

Ces inscriptions en calligraphie arabe permettent à l'auteur de faire passer des revendications et des messages politiques, d'exprimer son opinion. Le fait de les transmettre en arabe leur donne plus de légitimité car l' auteur ne passe pas par la langue du colonisateur. C'est un cri du cœur qui ne peut s'exprimer que par la langue arabe ${ }^{24}$.

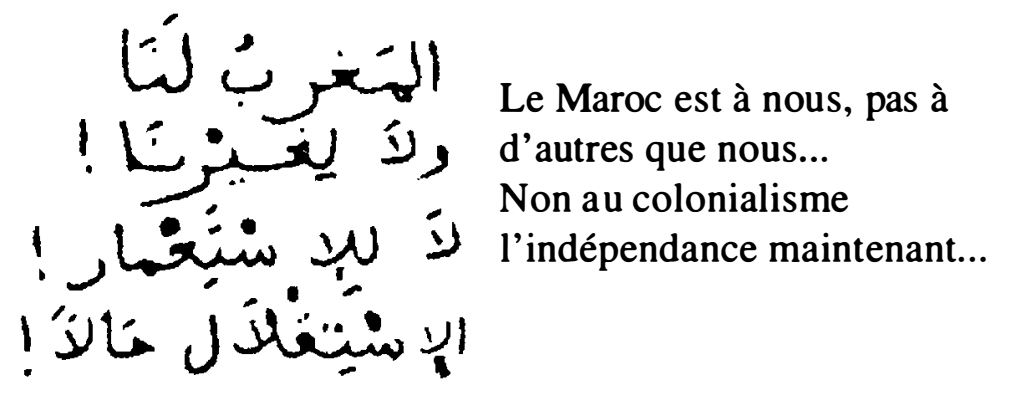

La beauté de la calligraphie fait rêver l'écrivain. Celle-ci ne fait qu'accentuer la grandeur du texte du Coran. Lorsqu'il est dans la mosquée, il se laisse emporter par la mélopée coranique créée par la récitation collective du Coran. Celle-ci l'élève vers les plafonds sculptés qu'il parcourt avec délice d'une lettre à une autre pour chevaucher :

$\overline{24}$ La Prière ..., 30-31. 
une belle prière :

- U Si Dieu vous donne la victoire,

personne ne peut vous vaincre.

Je m'accrochais au Alif et me laissais tirer par le Noun qui me déposait ans les bras du $\mathrm{Ba}^{25}$.

Des éléments essentiels et constitutifs de l'identité arabomusulmane sont l'Islam, la littérature, la philosophie. L'auteur en fait souvent référence car ils constituent un passé glorieux où les vertus et l'intelligence primaient.

Pour en parler, Ben Jelloun utilise la calligraphie afin de restituer toute leur beauté et leur pureté. En voici un exemple, il s'agit d'un vers d'Al Hallaj'26 :

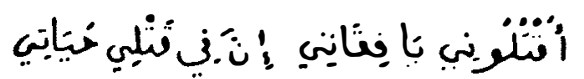

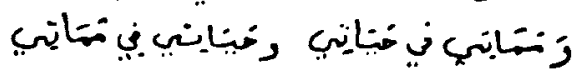

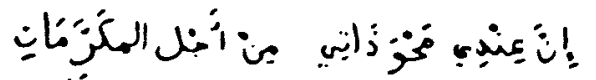

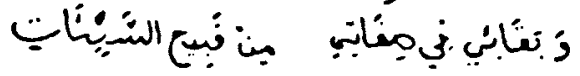
ي المسور البنالينلت

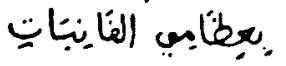
بني الفنز رالدًارئاتِ

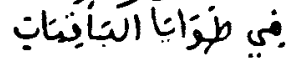
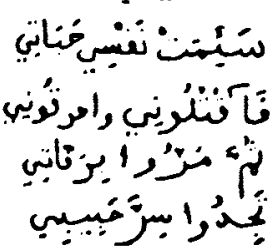

Tuez moi, ô mes amis

Ma vie est dans ma mort

Et ma mort est dans ma vie

Et ma vie c'est de mourir

Annuler mon être

C'est me faire un don

Me laisser tel que je suis

Est le pire des maux

Mon âme porte le dégoût de

/ma vie

A travers les décombres.

\section{Les références à l'Islam}

Dans l'œuvre de Tahar Ben Jelloun les références à l'Islam abondent. Elles manifestent la place primordiale de la religion dans 25 L'Enfant ..., 37.

26 La Prière ..., 193-194. 
la société musulmane. La foi des croyants est inébranlable : « ... notre enseignement est basé sur l'Islam. C' est notre culture et notre identité. Les Chrétiens le savent, c'est d'ici qu'est parti le mouvement pour l'Indépendance ${ }^{27}$.

L'écrivain présente les différents visages de l'Islam liés principalement au Coran : «le Livre fondateur d'une civilisation et qu'à ce titre on n'échappe pas à son emprise ${ }^{28}$ et au Prophète.

Il évoque des dates qui revêtent une gravité particulière pour les musulmans. En effet le père d'Ahmed meurt « la nuit du destin ", nuit sacrée qui correspond à la vingt-septième du mois de Ramadan. C'est la nuit du jugement où les « destins des êtres sont scellés » ${ }^{29}$ et pendant laquelle des anges viennent chercher les morts et mettre de l'ordre en toute chose. Les personnes doivent donc faire unexamende conscience, se confesser et essayer de réparer le mal qu' elles ont pu faire pendant leur vie. L'appel à la prière des muezzins rythme les heures qui passent. Hadj Ahmed, le père d'Ahmed/Zahra, a décidé d'élever sa huitième fille comme un garçon avec tous les avantages que leur octroie la société et la religion. La nuit du destin, il reconnaît l'hérésie qu'il a commise en essayant de détourner le destin d'Ahmed. Il l'affranchit de son terrible secret en lui conseillant de vivre selon sa vraie nature. Le personnage va tout abandonner et commencer un nouveau cycle de vie mais cette fois-ci en tant que femme.

Une autre date marquante qui ponctue la pratique religieuse islamique est le dixième jour du premier mois de l'Hégire (date du départ de Mahomet de La Mecque pour Médine). Elle remémore l'Achoura, anniversaire de l'assassinat du " petit-fils du prophète Mohammed Hossayn, fils de Ali Ibn Abi Tâlib et de Fâtima bint Mohammed ». Les enfants l'attendent avec impatience parce qu'ils reçoivent des cadeaux pendant que les adultes portent le deuil. Les

\footnotetext{
27 La Prière ..., 193.

${ }^{28}$ Laurence Khon-Pireaux, Tahar Ben Jelloun - L'Enfant de sable / La nuit sacrée (Ellipses : Paris, 2000) 59.

${ }^{29}$ La nuit sacrée, 23.
} 
places résonnent de musiques diverses et des conteurs rivalisent de créativité. Pendant ces deux célébrations religieuses, les prières occupent une place essentielle. Les prières rythment la vie des musulmans comme la Fatiha, "prière de la naissance, la prière qui donne le nom et fixe les racines de l'être ${ }^{30}$ ou la prière d'Al Fajr ${ }^{31}$. Outre les cinq prières de la journée, elles sont prononcées par la mère pour bénir les affaires de ses enfants. Ce sont les prières de la femme du patriarche (Moha le fou, Moha le sage) qui assurent la bonne fortune de son fils. C'est elle qui le guide en lui conseillant les prières à réciter pour invoquer l'aide d'Allah. Le grand père de Dada les psalmodie pour guérir les filles du village atteintes d'une folie soudaine.

Les préceptes, ou l'interprétation de ceux-ci, sont également rapportés à travers les commentaires des héros. Ceux qui sont cités soulignent, en particulier, l'injustice subie par les femmes. D'ailleurs, Moha dénonce la manipulation des règles de l'Islam faite par les hommes : «Il y a quelque chose de fêlé entre l'homme et la femme dans notre société. L'Islam. On dit que c'est dans le Livre Non. Ils font dire ce qu'ils veulent au Livre ${ }^{32}$.

D'autres références au Coran sont les sourates. Celles qui sont citées parl'écrivain favorisent les hommes en dépossédant les femmes ${ }^{33}$. D'ailleurs, le père d'Ahmed en souffrait car, n'ayant que des filles, tout son héritage, au moment de sa mort, serait partagé entre ses frères qui jubilaient de son malheur. Les Sourates : «Les Abeilles », «La Duperie », la Sourate IX, « Revenir de l'erreur ou l'Immunité », « Les impies " dénoncent les hypocrites et les impies parmi lesquels on compte également les extrémistes ${ }^{34}$.

\footnotetext{
${ }_{30}$ La nuit sacrée, 28.

31 La nuit sacrée, 192 ; Cf. également La nuit sacrée, 75.

32 Moha le fou ..., 47-48.

${ }^{33}$ L'Enfant ..., 52 - Sourate des femmes, IV, 11-12. Il est dit « Voici ce dont Allah vous fait commandement au sujet de vos enfants : au mâle, portion semblable à celle de deux filles *... ".

${ }^{34}$ Cf. Sourate * Les Abeilles ", Moha le fou, Moha le sage (163); Sourate « La Duperie » La Prière de l'absent (45). Il mentionne également les cent quatorze sourates du Coran. La Prière de l'absent (79), Sourate IX, «Revenir de l'erreur ou l'Immunité » L'Enfant de sable (170); Sourate " Les impies » (79), La nuit sacrée.
} 
Ben Jelloun accorde une grande importance à l'Islam parce que la littérature témoigne de la montée d'un certain extrémisme religieux dont il fait mention grâce à la sourate "Les impies »: « Nouveau regard porté sur un monde gagné par le réveil du fondamentalisme et ses comportements extrêmes ; ses appels souvent violents à un retour aux sources $»$.

La dérive amorcée par la religion islamique dans les pays arabes revêt une importance particulière pour Tahar Ben Jelloun. A travers ses personnages, il dévoile ce qui se passe dans les milieux religieux islamiques. Outre le vocabulaire déjà mentionné, il éparpille, le long des chapitres, des remarques, des récits et des mini-récits pour dénoncer un certain extrémisme. Il témoigne de la violence de plus en plus présente dans les mosquées. Dans Moha le fou, Moha le sage, l'enfant SDF raconte à Moha comment il a été abordé par un jeune fqih de la mosquée qui a tenté de l'embrigader dans son organisation pour espionner les gens et dénoncer ceux qui ne suivent pas les préceptes du Coran. Il met en garde le garçon contre la perte des valeurs islamiques et contre les étrangers qui ont une mauvaise influence sur le pays et sur les jeunes :

Il m'a longuement parlé de leur groupe. Ils sont quelques-uns comme lui à avoir formé un groupe de lutte pour la parole de Dieu. C' 'est un groupe armé de Corans et de poignards. Ils se réunissent souvent et discutent violemment entre eux. J'ai compris qu'ils recrutent leurs agents parmi la jeunesse. Ils se pointent à la sortie des lycées et font connaissance avec des jeunes gars... ${ }^{36}$

Dans La Prière de l'absent, le jeune fqih explique, avec beaucoup de passion et de violence contenues, les raisons qui poussent

${ }_{35}$ Tahar Bekri, Littérature de Tunisie et du Maghreb (Paris : L'Harmattan, 1994). 99.
${ }^{36}$ Moha le fou ..., 80. 
les gens à s'enrôler dans les groupes armés terroristes issus des milieux islamiques. En outre, dans le Coran, il est dit que les prophètes et les «martyrs » bénéficient d'un accès direct au paradis tandis que les autres morts ressuscitent le jour du jugement dernier. Ceci conforte la décision prise par toutes les personnes que l'humiliation et les discours des imans poussent à perpétrer des actes terroristes : "Casablanca est peut-être muette, mais elle n'est pas sourde. Elle accumule les réserves de passion et de violence, de colère et de révolte. Trop, trop d'humiliations. Le jeune fqih criait ${ }^{37}$.

L'implication politique de l'Islam est confirmée, à plusieurs reprises, par l'auteur à travers toute son œuvre.

Ben Jelloun évoque également dans Moha le fou, Moha le sage le «Madhi » personnage légendaire ou messie qui viendrait sauver la nation. Selon l'auteur ce serait un homme "ni héros ni martyr, un homme providentiel, sorte de prophète laïc, un sage épris de lucidité et de justice... », qui à l'instar de Soliman le magnifique unirait « la nation arabe et lui redonnerai une présence dans le monde et une dignité que le colonialisme a bafouée ${ }^{38}$.

Cependant, tout dans le Coran n'est pas négatif. Pour certains hommes doctes comme le Consul (La nuit sacrée) ou le troubadour aveugle (L'Enfant de sable) le Coran est une oeuvre empreinte d'une "sublime poésie ». Le troubadour aveugle, bien que n'étant pas musulman, admire la calligraphie et la beauté du Livre sacré des musulmans.

\section{Références à la littérature}

Expression d'un passé riche et raffiné, la littérature esquisse un autre visage de la culture arabo-musulmane. Outre la connotation poétique, esthétique et mystique elle connote une certaine nostalgie de

\footnotetext{
37 La Prière ..., 115.

38 Tahar Ben Jelloun, Le monde arabe est fatigué in Point de vue (Paris : Le Monde, 2003).
} 
l'auteur et, à travers lui, d'une certaine classe sociale éduquée d'un monde révolu : celui des grands poètes, écrivains, philosophes et mystiques. Ahmed / Zahra (L'Enfant de sable / La nuit sacrée), Sindibad, Jamal, les fqihs (La Prière de l'absent) connaissent les soufis des grands mystiques de l'Islam ${ }^{39}$. Ce courant considère que l'amour permet d'établir un contact spirituel intérieur avec Dieu, une fusion menant à l'illumination. C'est ce qui leur a valu la condamnation de l'orthodoxie ${ }^{40}$ qui croit à l'inaccessibilité de Dieu. L'oubli de soi est donc essentiel pour pouvoir rejoindre Allah. C'est probablement la raison pour laquelle Fatima, cousine et femme d'Ahmed, plonge dans la lecture des grands mystiques ${ }^{41}$ et oublie son corps malade qui emprisonne son âme. Elle se laisse peu à peu mourir pour suivre le chemin de la délivrance que les soufis lui ont montré. Le soufisme est très présent dans l'œuvre de cet écrivain. Sindibad, le jeune étudiant de bonne famille en est l'incarnation. Il avoue suivre les préceptes des grands mystiques qui détournent « l'esprit de la religion » selon le vieux cheikh de la Qaraouiyine (université) où il étudiait. Il a lu les textes de grands savants soufis Na't al-bidayat du cheikh Ma-alAynayn, récite des vers d'fiAl Hallaj, Hasan Basri, Ghazâli, Ibn 'Arabi et des poètes comme Abû Nawâss ${ }^{42}$. Ce détachement des soufis par rapport à la vie matérielle et au corps est palpable dans la description de ce personnage que l' auteur ébauche : « ...compagnies de la laine et du sang ", la laine du renoncement et le sang de la vérité, membre de la secte de l'Un ou disciple d'un maître des mots intérieurs ${ }^{43}$.

\footnotetext{
${ }^{39}$ Le nom soufis qui désigne ces mystiques de l'Islam dérive de souf ou çouf : la robe de laine blanche portée par les premiers mystiques. Cf. Encyclopédie multimédia Hachette. Ce courant apparaît dès le VIII $^{e}$ siècle à Koûf a et à Bassorah puis à Bagdad au IX ${ }^{\mathfrak{c}}$ siècle.

${ }^{40} \mathrm{Cf}$. Tahar Ben Jelloun confirme ce fait dans La Prière ..., 81 .

41 L'Enfant ..., 74.

${ }^{42}$ Parmi les soufis les plus connus Hasan al-Basri (mort en 772); Rabiyah. l'ancienne joueuse de flûte devenue sainte (morte en 801); Al-Djounayd (mort en 910): Al-Hallâj, qui connut le martyre à Bagdad en 922, ce qui témoigne de la rupture survenue entre l'orthodoxie et le soufisme; Al-Ghazali (10581111), qui parvint à inscrire le mysticisme dans l'orthodoxie; puis Djalal el-Din Roumi (mort en 1273) et l'Andalou Mohiyi ad-Din Ibn Arabi, sumommé Ech Cheikh al-Akbar ("le plus grand des maîtres spirituels ") (1165-1240).

${ }^{43}$ Moha le fou ...., 80.
} 
Ayant emmené l'enfant au terme de son initiation, il le rend à la femme enveloppée d'un halo de lumière qui va parfaire son éducation. Cette apparition de lumière n'est autre qu'Argane, la jeune fille mystérieuse qui les a accompagnés pendant leur périple vers le Sud. A l'instar de Fatima, son corps le lâche « sentit son corps partir lentement, comme si on retirait une peau, une fourrure ou un vêtement $"{ }^{44}$ pour n'être qu'esprit et fusion avec Allah. Le même sort est subi par Zahra (La nuit sacrée). Leur fin reste énigmatique. Ces personnages se retrouvent en présence de saints (Le Consul lui-même en est devenu un), d'êtres de lumière, d'initiés (Argane), des personnes mortes depuis longtemps mais qui ont retrouvé la paix (L'Assise). La fusion avec Allah tant cherchée par les soufis est palpable à la fin des diégèses. Ils se débarrassent d'un corps encombrant pour passer des ténèbres d'une vie terrible à un au delà plein de lumière où ils vont retrouver la paix. Le soufisme imprègne chaque ligne de la description faite. Le détachement du matérialisme ambiant est représenté par la djellaba d'homme en laine épaisse et rugueuse que porte Zahra, ou celle portée par le montagnard, le fqih qui accueille les pèlerins lors de leur première halte. La présence divine est suggérée par le décor : une lumière forte qui chasse la brume, les pierres ou les bâtiments blancs où séjournent des saints qu'on vient consulter pour s'apaiser. La lumière est omniprésente, elle est le symbole du divin tant dans le sens propre que dans le sens figuré. Et enfin l'amour, cet amour tant de Dieu que des hommes tant chanté par les soufis que Zahra retrouve avec le consul.

La littérature ne se borne pas aux textes des mystiques soufis, la poésie occupe une place très importante autant dans la culture arabe que dans l'œuvre de cet écrivain qui, rappelons-le, est aussi poète. Il s'est, en fait, essayé à tous les genres littéraires avec autant de maîtrise. Il a déjà publié cinq recueils de poésie. Celle-ci constelle les textes en prose à deux niveaux. Tout d'abord, l'auteur introduit, ici et là, des

44 La Prière ..., 232. 
commentaires : « L'amour ! Notre littérature, surtout la poésie, célèbre l'amour et le courage ${ }^{45}$, des vers de grands poètes comme Abû-lAlâ al-Ma' arrî, ou Abû Nawâss, évoque des recueils de poèmes tels que le Risalat al-Ghufran. Elle fait partie de la vie quotidienne d'un certain milieu éduqué dans lequel évoluent des Cheikhs, des érudits, des étudiants entre autres. Ils établissent parfois une sorte de rituel qui se répète inlassablement. Le cavalier qui enlève Zahra récite des vers d'Abû-l-Alâ al-Ma'arrî pour pouvoir entrer dans le village des enfants (La nuit sacrée). L'Assise, elle déclame également des vers pour réveiller son frère, le Consul, en toute douceur (La nuit sacrée). Sindibad est bercé par la musicalité des vers qu'il étudie à l'université (La Prière de l'absent). L'écriture foisonne de figures de style : des métaphores et des images propres au monde arabe telles que « J'ai la mémoire dans la plante des pieds ", " la nuit est une robe légère qui touche à peine les flots et les dunes ", "C'est une ville large comme un corps sans membres, un visage inachevé ", "La nuit est une prairie d"étoiles traversée de quelques murmures ${ }^{46}$. Force est de constater que les textes de Ben Jelloun regorgent d'anaphores, d'allitérations qui créent un rythme incantatoire lancinant visant à insister sur les manques, les absences des êtres. Il met en marche toute une machine stylistique avec d'autres procédés afin de représenter la déconstruction de toute une société. Ces procédés méritent d'être analysés en profondeur.

La lecture permet à tous les héros de s'évader d'une vie difficile à assumer, de survivre à des crises, de surpasser des états d'une profonde dépression. Lorsque leur souffrance est très forte ils s'enferment et passent des jours à lire. Après la mort de Fatima, Ahmed (L'Enfant de sable) se cloître dans sa chambre. Ses divagations s'enchaînent de livre en livre. Il s'amuse à imaginer leur auteur. Fatouma (L'Enfant de sable), elle aussi, avoua avoir passé des heures

${ }^{45}$ La nuit sacrée, 29.

46 L'Enfant ..., 156 ; La nuit ..., 129 ; La Prière ..., 113 ; Moha le fou .... 14. 
confinée dans une chambre ronde à imaginer des voyages infinis. La femme arabe rencontrée par le troubadour aveugle ( $L$ 'Enfant de sable) avait déjà beaucoup lu, lorsqu'elle le rencontre. Zahra et le Consul (La nuit sacrée) partagent ce plaisir de la lecture qui leur permet d'établir une relation d'amitié. En général, la lecture exerce une action bénéfique dans les héros. Cependant, elle peut être négative car elle détourne l'esprit des gens épris de connaissance comme dans le cas de Sindibad (La Prière de l'absent). Parmi les livres que ces protagonistes évoquent, les Mille et une nuits dont ils connaissent les contes, occupe une place de choix. Les plus grands personnages de l'histoire et de la littérature arabes y sont cités, mis en scène : «...califes abbassides et leurs vizirs d'origine persane, poètes, musiciens, joueurs d'échecs. Les références historiques s'ajustent en toute liberté à la légende » 47 .

Le conte est primordial dans l'œuvre de Tahar Ben Jelloun, tant au niveau de la narration qu'au niveau de la structure des romans. A l'instar des Mille et une nuits, l'auteur insère dans son œuvre différents types de contes allant du conte fantastique au conte « initiatique ${ }^{48}$ en passant par le conte merveilleux et la légende. D'ailleurs dans ces romans, il y a pléthore de conteurs chargés de transmettre les traditions au peuple illettré. Le fantastique et le merveilleux sont indissociables du monde arabe. Ils imprègnent autant l'espace physique et psychologique que fictionnel. Plusieurs récits fantastiques y sont insérés. La Prière de l'absent en compte dix. L'histoire même des protagonistes est en quelque sorte fantastique car ceux qui accompagnent l'enfant dans le voyage initiatique ne sont que des morts-vivants, en quelque sorte ressuscités, pour mener à bien leur mission. Une fois celle-ci accomplie leur apparence physique se désagrège pour ne faire qu'une brève apparition sous une lumière éblouissante. Dans La nuit sacrée, outre l'histoire de la maison

\footnotetext{
47 Cf. Encyclopédie multimédia Hachette.

48 Khon-Pireaux, 78.
} 
maudite racontée par l' Assise à Zahra, l'auteur y esquisse celles des personnages fantastiques tels que les djnouns ou les djinns de l'imagerie populaire. Les récits merveilleux sont moins nombreux. Leur fonction est de permettre au héros de prendre du recul, de s'y réfugier le temps de pouvoir appréhender sa nouvelle situation. C'est ainsi que se présente le récit du village des enfants. Telle une princesse, Zahra se fait enlever par un cavalier qui chevauche un cheval blanc. Celui-ci l'emmène dans un village que personne ne connaît et où n' habitent que des enfants. Celui qui s'y aventure perd peu à peu la mémoire, ce qui est essentiel pour Zahra qui veut se forger une nouvelle personnalité. D'autres histoires merveilleuses plongent le héros dans un univers linguistique et littéraire qui les protège des réalités cauchemardesques ou frustrantes de leur existence. C'est le cas du Consul qui découvre un hangar aux mots où les personnes empruntent les mots ou les phrases dont ils auront besoin dans la semaine ; et une bibliothèque où les livres sont récités par des superbes jeunes femmes.

Chaque panneau portait une lettre de l'alphabet. Ce hangar était un dépôt de mots. C'était le dictionnaire de la ville..., dissimulée dans le mur avec des étagères où sont déposés les mots cassés, abîmés ainsi que des mots anciens très usés et que personne n'utilise .... Là c'était différent. ...de jolies femmes qui apprenaient par cœur un roman, un conte ou une pièce de théâtre Cette bibliothèque humaine était très animé $e^{49}$.

Il est intéressant de remarquer que, dans ces contes fantastiques, l'écrivain insère d'autres mini-récits en contraposition pour accentuer l'importance que les livres ont pour lui et pour les arabes.

Outre la fonction dans l'œuvre elle-même, ces contes connotent l'appauvrissement de la langue ainsi que la perte d'intérêt des personnes pour la littérature. En ce qui concerne L'Enfant de sable, les métarécits

$\overline{49}$ La nuit sacrée ..., 23. 
féeriques connotent plutôt l'attachement de Ben Jelloun pour l'écriture et principalement pour la calligraphie arabe. Ils expliquent sa conception de la création littéraire. En effet, lorsqu'il était petit Ahmed, il accompagnait sa mère au hamman. Pendant que les femmes parlaient il laissait son imagination d'enfant l'emporter dans un monde imaginaire. Dans cet univers merveilleux, les mots et les lettres l'emmenaient dans un voyage, dont il ne connaissait pas la destination, à la recherche d'un discours cohérent dans le bavardage des femmes. Cette histoire connote évidemment une certaine misogynie qui dénie aux femmes l'intelligence de tout être humain. Enfin, d'autres contes reprennent des légendes qui appartiennent au monde arabe, celle du poete et guerrier Antar est reprise par l'auteur. Il a vécu quelques siècles avant l'Hégire et ses exploits ont inspiré des conteurs qui ont narré sa vie au cours des siècles. Une épopée chevaleresque : le Roman d'Antar, reprenant les différentes versions, est parue au XII siècle. Ben Jelloun en présente une version qui mélange les différents personnages associés à ce guerrier comme la femme-guerrière ou sa femme Abla qui n'hésite pas à tuer l'hommequi l' a capturée (Celle de Mahdi, entre autres). Ces récits fantastiques sont pris en charge non pas par un seul narrateur mais par plusieurs imposant ainsi une polyphonie narrative qui donne plus de vraisemblance aux contes puisqu'ils sont racontés comme de vraies histoires. Parfois une même histoire est narrée par deux rapporteurs, tactique qui permet à l'écrivain d'imposer dans l'esprit du lecteur une certaine véracité. Cette chaîne de conteurs anonymes est complétée par des conteurs professionnels.

Il y a deux catégories de conteurs. Tout d'abord, les vieux conteurs érudits et très religieux. En effet, ils ont la passion des livres, ils possèdent de grandes bibliothèques comme le troubadour aveugle qui a passé sa vie à lire jusqu'au moment où il a perdu la vue. Dans L'Enfant de sable ils sont au nombre de quatre. Il est remarquable le fait qu' ils sont anonymes, ils n'ont pas de nom. Ils sont connus comme « le vieux conteur » qui raconte l'enfance d'Ahmed, le troubadour aveugle, l'homme au turban et un autre qui n'est qu'une voix qui 
interpelle les auditeurs. On ne connaît leur nom que lorsqu'ils perdent leur prestige ; c'est ce qui arrive au vieil conteur de L'Enfant de sable. Ces conteurs sont très respectés par les auditeurs qui vont les suivre en signant un pacte de fidélité. Ils instaurent tout un rituel entre eux et leurs auditeurs qu'ils interpellent sans cesse. Ils utilisent tout d'abord une série de formules rituelles : "Ô mes compagnons, Ô mes amis, Amis et compagnons du Bien, Compagnons fidèles !, Ô gens de Bien », tout au long du récit pour maintenir le lien avec eux qui va se renforcer, au fur et à mesure, que l'histoire avance. Chaque jour, ils se réunissent dans une place publique pour un nouveau chapitre du récit. Ils s'y installent, étalent un tapis, s'assoient, les jambes croisées, et sortent un cahier ou un manuscrit qu'ils montrent à l'auditoire. Les conteurs terminent leur récit en posant des énigmes que leurs auditeurs doivent essayer de résoudre. Ils deviennent ainsi partie intégrante de l'histoire. Ces conteurs gardent ainsi les traditions orales de leur culture qu'ils enrichissent en ajoutant des détails, en modifiant, au cours des récits successifs, la trame pour donner naissance à d'autres histoires. En fait, ils s' approprient l' histoire des autres qu' ils modifient à leur gré ou à leur inspiration «... Sachez simplement que j'ai passé ma vie à falsifier ou altérer les histoires des autres... " ${ }^{50}$. Celle d'Antar le guerrier qui devient berger et femme de surcroît dans L'Enfant de sable, entre autres.

Ces conteurs entretiennent une relation quasi maladive avec leurs histoires souvent tirées d'un manuscrit ou d'un journal intime. Celles-ci les possèdent, les illuminent, les éblouissent au point d'en parler comme s'il s'agissait du livre sacré du Coran. Lorsqu'ils en prennent connaissance le texte est obscur. Une lecture ne suffit pas pour en saisir le sens. La description faite par le conteur le présente comme une perle rare provoquant des sensations aussi enivrantes que celles du Coran lui-même.

\footnotetext{
51) L'Enfant ..., 164.

51 L'Enfant ..., 12.
} 
J'ai été inondé par le parfum du paradis, un parfum tellement fort que j'ai failli suffoquer. J'ai lu la première phrase et je n'ai rien compris. J'ai lu le deuxième paragraphe et je n'ai rien compris. J'ai lu toute la première page et je fus illuminé... livre rare, le livre du secret, ....La lumière qui en émane éblouit et aveugle les yeux qui s'y posent par mégarde ${ }^{51}$.

D'ailleurs l'énigme de l'histoire est considérée un Secret sacré. La deuxième catégorie est constituée par de jeunes conteurs, la relève, en quelque sorte. Ils rivalisent avec les vieux conteurs qui perdent leur renommée à leur profit. Après un bref préambule l' histoire de La nuit sacrée débute par les récits des trois jeunes conteurs/ conteuses et du vieux conteur de L'Enfant de sable qui a perdu la mémoire à cause de l'histoire d'Ahmed. Leur inspiration leur vient d'une série d'objets banals qui peuvent aboutir en mille et une histoires. Ils se partagent les auditeurs qui s'agglutinent en cercle pour écouter leurs histoires. Ils n' ont pas encore le prestige ni le respect des vieux conteurs et peuvent être remis en question. Par la multiplication de conteurs, Tahar Ben Jelloun affirme son appartenance au monde arabe et par la même occasion son attachement aux traditions orales. D'après Laurance Kohn-Pireaux ${ }^{52}$, cette tradition est liée aux origines de l'Islam puisque les révélations reçues par le prophète Muhammad étaient orales et celui-ci, qui était illettré, va les transmettre oralement.

Curieusement, dans La Prière de l'absent il n'y a que quatre conteurs professionnels. Ils ont pour fonction de rappeler l'importance de l'oralité ainsi que d'introduire l'élément fantastique qui marque fortement la tradition arabe.

Finalement, on peut affirmer que Tahar Ben Jelloun met en place tout un réseau lexical dénotatif grâce auquel, il dépeint une culture possédant un passé glorieux que des sages, des philosophes, des mystiques, des héros, des poètes ont porté à leur apogée. Les vertus

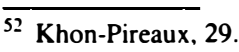


de l'Islam ont façonné des héros qui ont défendu le monde arabe contre des envahisseurs occidentaux. Leurs exploits ont été transmis aux générations par des conteurs dans les places publiques en insufflant la fierté à tout un peuple. La perte des valeurs, les humiliations subies à travers l'histoire engendrent des sentiments de haine que certains religieux exploitent et qui provoquent des réactions violentes et un sentiment anti-occidental dont on constate les conséquences aujourd'hui.

Bien qu'écrivant ses romans en français, c'est le Maroc et la culture arabo-musulmane qui s'impose dans ses récits dont la présence est incontournable. L'Islam, qui constitue le cœur même de cette culture, s'égrène le long des textes permettant au lecteur non averti de comprendre un peu mieux la religion et les croyances des peuples musulmans. La littérature souligne en particulier le réveil du fondamentalisme avec ses comportements extrêmes, ses appels souvent violents à un retour aux sources.

L'auteur réussit une peinture très réaliste de la culture arabomusulmane avec son passé prestigieux et son présent qui est un mélange de modernité et de Moyen Age, en particulier, en ce qui concerne la situation de la femme et les déshérités. 